\title{
Assessing nitrous oxide effect using electroencephalographically-based depth of anesthesia measures cortical state and cortical input
}

Levin Kuhlmann ${ }^{1,2, *}$ and David T J Liley ${ }^{1}$

1. Brain and Psychological Sciences Research Centre, Swinburne University of Technology, PO Box 218, Hawthorn VIC 3122, Australia

2. NeuroEngineering Laboratory, Department of Electrical and Electronic Engineering, The University of Melbourne, Melbourne, Victoria, Australia.

* Corresponding author

Corresponding author contact details: Ph - +61 41255 2283; Fax - +61 39214 4361;

Email: levink@unimelb.edu.au

\section{Acknowledgements}

This work was financially supported by Swinburne University of Technology intramural funds, ARC Linkage Grant LP120200773, and Cortical Dynamics Ltd. We thank Brett Foster from Stanford University for contributing to the original collection of the dataset and Denny Meyer from Swinburne University of Technology for her statistical analysis advice. 


\begin{abstract}
Purpose: Existing electroencephalography (EEG) based depth of anesthesia monitors cannot reliably track sedative or anesthetic states during n-methyl-d-aspartate (NMDA) receptor antagonist based anesthesia with ketamine or nitrous oxide $\left(\mathrm{N}_{2} \mathrm{O}\right)$. Here, a physiologicallymotivated depth of anesthesia monitoring algorithm based on autoregressive-moving-average (ARMA) modeling and derivative measures of interest, Cortical State (CS) and Cortical Input (CI), is retrospectively applied in an exploratory manner to the NMDA receptor antagonist $\mathrm{N}_{2} \mathrm{O}$, an adjuvant anesthetic gas used in clinical practice.
\end{abstract}

Methods: Composite Cortical State (CCS) and Composite Cortical State distance (CCSd), two new modifications of CS, along with CS and CI were evaluated on electroencephalographic (EEG) data of healthy control individuals undergoing $\mathrm{N}_{2} \mathrm{O}$ inhalation up to equilibrated peak gas concentrations of 20,40 or $60 \% \mathrm{~N}_{2} \mathrm{O} / \mathrm{O}_{2}$. In particular, CCSd has been devised to vary consistently for increasing levels of anesthetic concentration independent of the anesthetic's microscopic mode of action for both $\mathrm{N}_{2} \mathrm{O}$ and propofol.

Results: The strongest effects were observed for the $60 \%$ peak gas concentration group. For the 50-60\% peak gas levels, individuals showed statistically significant reductions in responsiveness compared to rest, and across the group CS and CCS increased by $39 \%$ and $42 \%$, respectively, while CCSd was found to decrease by $398 \%$. On the other hand a clear conclusion regarding the changes in CI could not be reached.

Conclusions: These results indicate that, contrary to previous depth of anesthesia monitoring measures, the CS, CCS, and especially CCSd measures derived from frontal EEG are potentially useful for differentiating gas concentration and responsiveness levels in people under $\mathrm{N}_{2} \mathrm{O}$. On the other hand, determining the utility of $\mathrm{CI}$ in this regard will require larger sample sizes and potentially higher gas concentrations. Future work will assess the sensitivity of CS-based and CI measures to other anesthetics and their utility in a clinical environment. 
Keywords: autoregressive moving-average modelling, nitrous oxide, sedation, anesthesia, electroencephalography.

\section{Introduction}

The most widely used EEG-based automated depth of anesthesia monitoring system is the Bispectral Index (BIS) monitor (Medtronic, Dublin, Ireland) [1,2]. A recent clinical trial demonstrated that the BIS monitor was no more effective at preventing intraoperative awareness or post-operative recall, than knowledge of the anesthetic concentrations [3]. In a comprehensive review of this clinical trial and other related clinical studies it was found that use of the BIS monitor reduced the use of anesthetics, decreased recovery times, and shortened the duration of post-anesthesia care unit stay $[4,5]$. Therefore although there are benefits to using EEG-based automated depth of anesthesia monitoring, improvements are still needed. Moreover, a reliable depth of anesthesia monitor for use with the putative n-methyl-D-aspartate (NMDA) receptor antagonists ketamine and nitrous oxide $\left(\mathrm{N}_{2} \mathrm{O}\right)$ needs to be developed [6].

With regards to $\mathrm{N}_{2} \mathrm{O}$, in one study $\mathrm{N}_{2} \mathrm{O} / \mathrm{O}_{2}$ levels up to $50 \%$ caused no change in BIS and no obvious reductions in responsiveness as assessed by the observers' assessment of alertness/sedation (OAA/S) scale [7]. However, in another study at higher levels of $70 \% \mathrm{~N}_{2} \mathrm{O} / \mathrm{O}_{2}$ the BIS was not sensitive to $\mathrm{N}_{2} \mathrm{O}$ induced reductions in responsiveness [8]. The BIS also shows inconsistent changes when $\mathrm{N}_{2} \mathrm{O}$ is combined with other anesthetics. The BIS increases when $\mathrm{N}_{2} \mathrm{O}$ is combined with isoflurane [9], but decreases when $\mathrm{N}_{2} \mathrm{O}$ is added to propofol and sufentanil initiated, and sevoflurane maintained anesthesia [10]. Other depth of anesthesia monitors also show similar problems [11-13]. For example, entropy measures (M-Entropy plug-in Module S/5; Datex-Ohmeda Division, Instrumentarium Corporation, Helsinki, Finland) [12] and the Cerebral State Index ${ }^{\mathrm{TM}}$ (CSITM, Danmeter, Odense, Denmark) [11] demonstrated no change for $\mathrm{N}_{2} \mathrm{O}$ levels of up to $75 \%$ (with premedication of $30 \mathrm{mg}$ i.v. propofol) for which loss of consciousness 
was observed using the OAA/S score. These results are potentially due to a focus on characterising the effects of $\gamma$-amino-butyric-acid (GABA)-receptor based anesthetics on the EEG, and a lack of consideration of NMDA-receptor-based anesthetics [6]. Therefore, further work is needed to improve depth of anesthesia monitoring devices, in particular with regard to NMDA receptor antagonist based anesthesia and more generally to the clinical reality of poly-pharmacy.

Recently, the Brain Anesthesia Response (BAR) monitor (Cortical Dynamics, North Perth, WA, Australia) has been developed. The BAR monitor algorithm is based on fixed-order ARMA modeling [14,15], namely the Cortical State (CS) and Cortical Input (CI) measures derived from ARMA models, and represents a physiologically motivated approach to depth of anesthesia monitoring as the ARMA models can be mathematically related to models of cerebrocortical neural population activity [16-18]. These neural population models represent the inner workings of cerebral cortex and offer the potential to gain insights into anesthetic-induced neurophysiological parameter changes, for example by enabling estimation of propofol-induced inhibitory post-synaptic potential changes [19]. The use of neural models in the context of depth of sedation/anesthesia monitoring is a developing field and users should be aware that they represent an approximation to the relevant real brain regions being modelled [16-19]. Here we focus purely on ARMA models and their derived measures, and do not consider any direct relationship to neural models of cortical activity.

The BAR monitor measures have been shown to be able to dissociate the effects that hypnotic and analgesic agents have on brain electrical activity [18]. The intuition behind the CI measure is that sub-cortical sensory and nociceptive input is reduced by analgesics, while the CS measure characterizes the effects of hypnotic agents on cortical activity [16-18]. To date the CS and CI indices have been applied to the simultaneous application of the agents propofol and remifentanil [18], and sevoflurane and $\mathrm{N}_{2} \mathrm{O}$ [17] in the clinical setting. For example, for 
simultaneous propofol and remifentanil induction, it was found that CS decreases with increases in the effect-site concentration of propofol and concomitant reductions in consciousness, while CI remains unchanged [18]. In contrast, CI was found to decrease with increases in the effect-site concentration of the ultra-short acting synthetic opioid remifentanil [18].

The CS measure, and its novel derivative measures Composite Cortical State (CCS) and Composite Cortical State distance (CCSd) considered here, reflect the dominant damped (dissipative) oscillations present in the EEG power spectrum determined via the ARMA model, or in other less precise terms, the dominant time-varying oscillations in the EEG. The CI measure reflects the power of the estimated input to cerebral cortex as approximated by the ARMA model [16-18].

For the purposes of further understanding how the BAR monitor indices, CS, CCS, CCSd and CI, are effected by different substances, these measures are applied to EEG data obtained from healthy controls undergoing $\mathrm{N}_{2} \mathrm{O}$ inhalation. The putative NMDA receptor antagonist, strong sedative and weak anesthetic, $\mathrm{N}_{2} \mathrm{O},[20-24]$ provides an interesting contrast to the $\mathrm{GABA}_{\mathrm{A}}$ receptor agonists, such as propofol and sevoflurane [25]. Studying $\mathrm{N}_{2} \mathrm{O}$ alone is scientifically interesting because it provides us with a tool to investigate intermediate states of drug induced reductions in consciousness, as well as different pharmacological pathways of sedation/hypnosis. Clinically, it is interesting because as an adjuvant gas it is often first applied before a more potent anesthetic (e.g. sevoflurane) is added, and it is still important to monitor depth of anesthesia/sedation at this initial stage to ensure the more potent anesthetic is not over supplied [21].

\section{Methods}

Ethics approval for this study was obtained from the Swinburne University of Technology Human Research Ethics Committee in accordance with the Declaration of Helsinki. Informed written consent was obtained from each of the twenty healthy human males (between the ages of 18-40) that were recruited. 


\section{EEG dataset}

EEG data was acquired in a noise-minimised laboratory. The EEG dataset obtained during $\mathrm{N}_{2} \mathrm{O}$ inhalation that is studied here, has been analysed in prior studies on spectral power [20], topography [26], and functional connectivity networks [27]. Here we apply the ARMA measures, CS, CCS, CCSd and CI to a single frontal channel, FPz referenced to a linked mastoids reference (similar to standard clinical practice), from this dataset in order to assess the capacity of these measures to track gas concentration and depth of sedation during $\mathrm{N}_{2} \mathrm{O}$ inhalation. The details of the dataset are published elsewhere [20]. Here the main aspects are summarised. Individuals underwent two EEG recordings: a five minute eyes closed rest recording and a twenty-minute gas recording. The twenty-minute gas recording involved a five-minute equilibration period, a 10 minute equilibrated peak $\mathrm{N}_{2} \mathrm{O}$ gas period, followed by a five-minute $\mathrm{O}_{2}$ only washout period. In the subsequent analysis only the first fifteen minutes of each gas recording was considered. Each individual experienced only one peak gas level. The peak gas levels of 20,40 or $60 \% \mathrm{~N}_{2} \mathrm{O} / \mathrm{O}_{2}$ were experienced by eight, eight and four individuals, respectively. The smaller number in the $60 \%$ group was related to issues with nausea and emesis. End-tidal gas concentration was monitored continuously. EEG data was acquired with a 64 channel extended 10-20 montage at $500 \mathrm{~Hz}$ with a $0.1-70 \mathrm{~Hz}$ bandpass anti-aliasing filter. EEG segments containing artifact were rejected based on visual inspection. Artifact-rejected EEG was subsequently band-pass filtered between 1 and $40 \mathrm{~Hz}$. Any remaining artifact such as eye blinks and electromyograph (EMG) were successfully removed using independent-component analysis as implemented in the EEGLAB toolbox [28]. As mentioned above data analysis focused on the $\mathrm{FPz}$ channel with a linked mastoids reference. Participant responsiveness was assessed as a means to indirectly assess level of sedation. In order to assess responsiveness, during the rest and gas recordings participants performed an auditory continuous performance task (aCPT) [20]. In the aCPT the participant had to press either a left or right button if a 1 or $2 \mathrm{kHz}$ tone was heard, respectively. Accuracy

\section{Page 6}


(correct/incorrect) was logged as a measure of responsiveness, where 'incorrect' also refers to missed trials.

\section{Pre-processing}

EEG data was resampled from 500 to $80 \mathrm{~Hz}$ using the built in 'resample' function in MATLAB (MathWorks, Natick, MA) which automatically generated and applied a 5001-order $40 \mathrm{~Hz}$ lowpass anti-aliasing finite impulse response filter with $60 \mathrm{~dB}$ attenuation at $40.5 \mathrm{~Hz}$. Amplitude spectra were visualized after resampling to ensure aliasing effects were minimized. The EEG signal was broken into 2 second epochs with $50 \%$ overlap. An epoch was rejected if the rootmean-squared (RMS) power of the mean-removed signal was greater than $150 \mu \mathrm{V}$. In order to relate the $\mathrm{CS}$ and $\mathrm{CI}$ measures to $\mathrm{N}_{2} \mathrm{O}$ gas concentration and responsiveness, linear and nearest neighbor interpolation were applied to sample the $\mathrm{N}_{2} \mathrm{O}$ gas concentration and aCPT accuracy time series at the start times of the EEG windows, respectively.

\section{Measure Analysis}

The CS, CCS, CCSd, CI, two other measures considered below (pole damping and pole frequency) and RMS power (as a control) were computed on the preprocessed and accepted 2 second epochs, and the measures' time-series were subsequently related to $\mathrm{N}_{2} \mathrm{O}$ gas concentration and responsiveness. To better understand the relationship between changes in the above measures and EEG frequency changes, we also considered power in the frequency bands of 1-4 Hz $(\delta), 4-8$ $\mathrm{Hz}(\theta), 8-15 \mathrm{~Hz}(\alpha), 15-30 \mathrm{~Hz}(\beta)$, and $30-110 \mathrm{~Hz}$ not including line noise at $50 \mathrm{~Hz}$ and $100 \mathrm{~Hz}$ ( $\gamma$, high $\gamma$ and overlapping EMG [29]). Although EMG can sometimes occur at frequencies below $30 \mathrm{~Hz}$ [30], given EMG's broadband nature if it is present below $30 \mathrm{~Hz}$ it will also most likely be present in the $30-110 \mathrm{~Hz}$ band [31]. Thus the $30-110 \mathrm{~Hz}$ band acts as a way of checking for existence of EMG. 
ARMA Analysis

The ARMA analysis methods applied here have, apart from the new CCS and CCSd measures, been presented in detail elsewhere $[17,18]$. Briefly we define how the CS, CCS, CCSd and CI measures are obtained. ARMA modeling with an autoregressive order of 8 and a moving average order of 5 is applied to approximate the transfer function of a linearized version of a nonlinear mathematical model of neural population activity underlying the EEG signal and influenced by anesthesia [17]. Thus the sampled EEG signal $s[n]$ (samples indexed by $n$ ) was modelled using an $(8,5)$ ARMA model

$s[n]=-\sum_{k=1}^{k=8} a_{k} s[n-k]+\sum_{l=0}^{l=5} b_{l} u[n-l]$

or

$A(z) S(z)=B(z) U(z)$

where $u[n]$ represents a stationary white noise process, $a_{k}$ and $b_{l}$ are the respective autoregressive and moving average parameters, all of which are estimated using algorithms described elsewhere $[14,15,18]$. The dummy variable $k$ indexes the autoregressive parameters $a_{k}$ from 1 up to (the autoregressive order of) 8 . The dummy variable $l$ indexes the moving average parameters $b_{l}$ from 0 up to (the moving average order of) $5 . S(z)$ and $U(z)$ are the Ztransforms of $s[n]$ and $u[n]$, respectively, where $z$ is, in general, a complex number and

$$
A(z)=1+\sum_{k=1}^{k=8} a_{k} z^{-k}
$$

and

$$
B(z)=\sum_{l=0}^{l=5} b_{l} z^{-l}
$$

The function $B(z) / A(z)$ represents the electrocortical filter and describes how subcortical input, $U(z)$, is filtered to give rise to the EEG. The poles, $\rho_{k}$, and zeros, $Z_{l}$, of the electrocortical 
filter are the solutions to $A(z)=0$ and $B(z)=0$, respectively. Given that $z$ is complex, the poles and zeros are also complex numbers. The poles essentially correspond to the dominant oscillations in the signal or the peaks in the power spectrum of the signal, while the zeros essentially indicate frequency points in the power spectrum where no power is present. Given that $A(z)$ and $B(z)$ are $8^{\text {th }}$ and $5^{\text {th }}$ order polynomials, there are therefore 8 solutions to $A(z)=0$ (i.e. 8 poles) and 5 solutions to $B(z)=0$ (i.e. 5 zeros), respectively. For a given ARMA model estimated from a given window of data, the original CS measure is the negative of the scaled mean pole projected onto the real axis, or equivalently

$\mathrm{CS}=\frac{a_{1}}{8}$

The novel, noise robust CCS measure is the negative of the scaled mean pole minus the mean zero projected onto the real axis, or equivalently

$\mathrm{CCS}=\frac{\left(a_{1}-b_{1}\right)}{13}$

This CCS measure has recently been demonstrated to be robust to noise for propofol anesthesia [32].

The CCSd measure is defined as the negative of the normalized distance between the current CCS value and the median CCS value obtained in the resting state, $\mathrm{CCS}_{\text {rest }}$, as follows

$\mathrm{CCSd}=-\frac{\left|\mathrm{CCS}-\mathrm{CCS}_{\text {rest }}\right|}{\left|\mathrm{CCS}_{\text {rest }}\right|}$

The CCSd measure is normalized relative to rest and made negative in order to define a CS-based measure that extends our prior work by providing a measure that consistently decreases with increases in anesthetic level for both $\mathrm{N}_{2} \mathrm{O}$ and propofol when each drug is applied separately. Our previous work applying CS to propofol found a decrease in CS with increasing anesthetic concentration [18]. Because a priori we do not know if CS increases or decreases with $\mathrm{N}_{2} \mathrm{O}$ gas concentration we therefore chose to devise CCSd, a measure that will decrease with increases in 
either propofol or $\mathrm{N}_{2} \mathrm{O}$ concentration, and thus provide a measure that varies in the same direction as a function of concentration and responsiveness for the anesthetics considered. We do not normalize the other measures described in this section as we are primarily seeking to advance the CS based measures for the aforementioned reasons.

The CI measure is the square root of the variance of $Z^{-1}(A(z) S(z) / B(z)$ ) (i.e. the estimated input). This is the same definition for CI that has been applied to propofol and remifentanil previously [18].

In order to better understand how $\mathrm{N}_{2} \mathrm{O}$ induced changes in CS/CCS/CCSd emerge, the damping (i.e. degree of dissipation) and frequency of the dominant oscillations were also considered. For a given pole, $\rho$, the damping and frequency of the oscillation described by the pole are $\gamma=-F_{s} \ln |\rho|$ and $f=\left(F_{s} / 2 \pi\right) \arg (\operatorname{Im}(\rho) / \operatorname{Re}(\rho))$, respectively, where $F_{s}$ is the data sampling rate. Given that the pole, $\rho$, is complex it can be noted that the damping, $\gamma$, is a nonlinear function of the modulus of the pole (i.e. the distance of the pole relative to the origin in the complex plane) while the frequency, $f$, is linearly related to the argument of the pole (i.e. the angle of the pole relative to the positive real axis in the complex plane). The more negative the damping parameter, $\gamma$, the greater the damping of the corresponding oscillation (i.e. a single frequency decays as $\exp [\gamma t])$.

\section{Statistical Methodology}

Given the small number of subjects in the $60 \%$ peak gas group, a detailed statistical analysis was applied to obtain multiple perspectives of the data. Moreover, given that the data being analysed were time series (i.e. many repeated measures), the individuals' measure (i.e. CS, $\mathrm{CI}$, etc) time series were processed using a standard non-parametric approach for quantifying the performance of depth of anesthesia monitoring measures called prediction probability [33] $\left(P_{K}\right)$. Prediction probability is also useful for quantitatively assessing and comparing the ability of the measures to indicate the $\mathrm{N}_{2} \mathrm{O}$ gas concentration level. 
Prediction probability is essentially a measure of correlation that works well for nonlinear monotonic relationships between two variables, in this case the measure level and the anesthetic concentration level. The simpler Pearson's correlation coefficient would not be an adequate alternative for our purposes as it only assesses linear correlations and the measures we consider typically have a nonlinear dependence on anesthetic concentration in general [18]. For the case of this paper we consider that the ideal depth of anesthesia measure is expected to have a perfectly monotonic relationship with anesthetic concentration. If there is no correlation between the measure level and the anaesthetic concentration then $P_{K}=0.5$. Furthermore, for measures that appeared to increase with increases in anesthetic concentration it was defined that if the measure level increases with a perfectly monotonic relationship with increases in anesthetic concentration level, then $P_{K}=1$. Similarly, for measures that appear to decrease with increases in anesthetic concentration we define that if the measure level decreases with a perfectly monotonic relationship with increases in anesthetic concentration level, then $P_{K}=1$. This enabled direct comparison of the $P_{K}$ estimates for the different measures regardless of the direction of change of the measures with increases in gas concentration. Prediction probability $P_{K}$ values approaching 0 indicate anti-correlation with respect to the direction of change of a measure. The direction of change of a measure was determined as the direction that gave the maximal mean prediction probability across the subjects in the $60 \%$ peak gas group when either of two cases were considered: the measure (1) increases or (2) decreases with increases in gas concentration. Prediction probability was computed using methods published elsewhere [33]. To compute the prediction probability one needs to relate a given measure to the simultaneously measured endtidal $\mathrm{N}_{2} \mathrm{O}$ gas concentration. For individuals in each peak $\mathrm{N}_{2} \mathrm{O}$ gas group, $20 \%, 40 \%$ and $60 \%$, this was achieved by binning measure values from each measure time series into seven bins, one for rest and six equal-sized, non-overlapping bins spanning the range of measured $\mathrm{N}_{2} \mathrm{O}$ gas levels in each group: $0-20 \%, 0-40 \%$ and $0-60 \%$ for the $20 \%, 40 \%$ and $60 \%$ peak gas groups, 
respectively. Given that not all bins for an individual contained the same number of measure samples, a resampling procedure was used to estimate the prediction probability and correct for this sampling bias. The procedure involved resampling the bins 100 times while ensuring the number of samples selected from the non-empty bins was always equal. The mean of the prediction probability across these 100 samples was considered the prediction probability estimate.

Given that prediction probability values closer to 1 indicate monotonic signals, the prediction probability also doubles as a non-parametric way to detect trends (i.e. increases or decreases) in signals. To statistically assess if prediction probability estimates show a significant difference from the prediction probability of an uncorrelated measure-gas level relationship, $P_{K}=0.5$, and thus are indicative of trends, a surrogate testing method was employed. For each individual for each of the 100 datasets used to estimate prediction probability, 50 different uncorrelated surrogate datasets were randomly generated where the mean of each rest or gas bin was set to zero and the standard deviation of each gas bin matched the standard deviation of the gas bin for the corresponding real data. For each of these 5000 surrogate datasets (50 surrogate datasets for each of the 100 original datasets) the prediction probability was calculated. To assess if the prediction probability estimate for each individual was statistically significant a MannWhitney U test [34] was used to compare the original and the surrogate datasets. This nonparametric test was employed because prediction probability takes values between 0 and 1 and thus any distribution of prediction probability will not have infinite tails like a Gaussian distribution.

At the group level, the prediction probability estimates for all 20 individuals were assessed in two ways. First a multivariate general linear model [35] with all measures selected as dependent variables and peak gas group treated as a fixed factor was used to statistically assess differences between the peak gas groups. This required the assumption that prediction 
probabilities estimated from subjects within a measure and within a peak gas group were normally distributed. Assessing the differences for each measure across the peak gas groups was done by testing for multiple comparisons using the student-Neumann-Keuls test [36]. Second a repeated measures general linear model [37] with the 7 measures set as within-subject factors was used to assess the difference of the prediction probability estimates for the measures. In particular, given that CCSd is the main measure of interest, a simple contrast was applied using CCSd as the reference for testing against the other measures. This model was applied to all peak gas groups combined since there were not enough subjects in the $60 \%$ peak gas group to have enough statistical power to analyse each peak gas group separately.

Furthermore, to statistically assess the differences in the measures in the gas level bins compared to the rest bin at the group level a linear mixed model [38] was applied to each measure (including power in the five frequency bands) within each peak gas group. First for each individual the mean and standard deviation of each measure in the rest and 0-10\%, 10-20\%, 20$30 \%, 30-40 \%, 40-50 \%$ and $50-60 \% \mathrm{~N}_{2} \mathrm{O}$ gas concentration bins were determined. Then the mean measures in each bin were combined across the individuals to determine the mean and standard deviation in each bin at the group level. A mixed model was applied because not all subjects had data in each gas bin. The mixed model treated $\mathrm{N}_{2} \mathrm{O}$ gas concentration as a repeated measure with a repeated covariance type of $\mathrm{AR}(1)$ dependence. Normality of the mean measure values within each bin and across subjects was assumed. Separate models were created for each measure, with each measure treated as the dependent variable and the gas concentration treated as a fixed factor. Model parameters were estimated using restricted maximum likelihood [38].

For all statistical tests that involved linear mixed models or general linear models, significance levels of $\alpha=0.05$ or 0.01 were applied.

To indicate that the measure dependence on gas concentration also meant a measure dependence on responsiveness, the relationship between gas concentration and aCPT accuracy was also determined. 


\section{Results}

Before considering the group results we consider the data from individuals in the $60 \% \mathrm{~N}_{2} \mathrm{O}$ peak gas concentration group because they showed the strongest and most consistent effects. Moreover, it is important to see data from individuals as in the clinical setting these methods would be applied on an individual basis.

Example EEG data for an individual in the $60 \%$ group

To provide an idea of what the EEG data looks like during significant $\mathrm{N}_{2} \mathrm{O}$ inhalation, Figure 1 illustrates example EEG data from an individual in the $60 \%$ peak gas group (subject 17) observed during the (A) baseline resting eyes closed period and during the (B) equilibrated peak level of $60 \% \mathrm{~N}_{2} \mathrm{O}$ gas concentration. The corresponding amplitude spectra for (C) baseline and (D) gas are also presented. It can be noted that this particular subject's EEG undergoes a clear reduction in amplitude in the $0-5 \mathrm{~Hz}$ and $8-12 \mathrm{~Hz}$ bands when comparing baseline to peak gas of $60 \% \mathrm{~N}_{2} \mathrm{O}$ gas concentration.

Measure dependence on concentration for individuals in the $60 \%$ group

For the four individuals in the $60 \% \mathrm{~N}_{2} \mathrm{O}$ peak gas group, each subfigure in Figure 2 shows the mean and standard deviation of a given measure for each rest and $\mathrm{N}_{2} \mathrm{O}$ gas concentration bin for (A) CS, (B) CCS, (C) CCSd, (D) CI, (E) the pole frequency $f$ in the 5-15 $\mathrm{Hz}$ band, (F) the pole damping $\gamma$ in the $5-15 \mathrm{~Hz}$ band, and (G) RMS power. (The pole frequency and damping were analysed in the $5-15 \mathrm{~Hz}$ band because of the strong damping seen in this band previously by Yamamura et al. [39] and as visualised here in Appendix Figure A1). Surrogate testing of the prediction probability estimates $P_{K}$ for all measures revealed that all subjects in the $60 \% \mathrm{~N}_{2} \mathrm{O}$ peak gas group, except for subject 17 for pole frequency, showed prediction probability estimates were significantly different from the uncorrelated case, $P_{K}=0.5(p<0.01)$. Figure 2(H) shows the number of epochs contributed by each subject to each gas bin. 
In Figure 2 it can be observed that CS and CCS increase and CCSd and RMS power decrease with increases in $\mathrm{N}_{2} \mathrm{O}$ gas concentration, while $\mathrm{CI}$ is highly variable, pole frequency has only very weak correlations with gas level and the pole damping parameter $\gamma$ shows decreases above $40 \%$ gas concentrations but only for some subjects. Subject 17 (red) had missing data for the $0-10 \%$ and $10-20 \%$ gas concentration bins due to rapid gas delivery for this subject, however, it is the higher gas levels that show the strongest effects in the $60 \%$ gas group. Due to nausea and emesis, Subjects 18 (green) and 19 (blue) had their gas recordings shortened to less than 5 and 10 minutes in duration, respectively. Thus subject 18 had missing data for the $50-60 \%$ gas bin. Despite the shortened recordings these two subjects still experienced a loss of responsiveness at high gas levels. For the data shown in Figure 2 the corresponding aCPT accuracy for subjects 17, 18, 19 and 20 during rest was $100,95.2,94.4$, and $96.8 \%$, respectively, and during the last two minutes of the gas recording was $0,0,0,88.4 \%$, respectively. The relatively high performance for subject 20 at the peak gas level, although still a reduction in responsiveness and performance, can be understood in terms of the varied and dissociative effects of $\mathrm{N}_{2} \mathrm{O}$ on individuals [40].

\section{Prediction probability estimates for all subjects}

The complete set of prediction probability estimates $P_{K}$ for each feature for each individual and the mean in the 20,40 and 60\% peak gas groups are displayed in Table 1. Generally, the greater the difference between a prediction probability value and 0.5 (the zero correlation case) the greater the degree of monotonicity in the relationship between a given measure and gas concentration [41]. The table highlights in italics the prediction probability estimates that were statistically significantly different from the uncorrelated case based on surrogate testing $(p<0.01)$ and also showed positive correlation with respect to the direction of change of the measure (i.e. $P_{K}>0.5$ ). It can be observed that the prediction probability estimates can be highly variable, reflecting the different subjects' sensitivities to $\mathrm{N}_{2} \mathrm{O}$. Moreover 
statistically significant prediction probability estimates with $P_{K}>0.5$ are most consistently present for the $60 \%$ peak gas group, indicating that this gas level had the greatest effect.

This is also reflected in the mean prediction probabilities of each gas group. For the $20 \%$ peak gas group, the highest mean prediction probability was $P_{K}=0.59$ and this was for CCSd. For the $40 \%$ peak gas group, the highest mean prediction probability was $P_{K}=0.62$ and this was for CI, while for the primary measure of interest, CCSd, it was $P_{K}=0.61$. For the $60 \%$ peak gas group, the highest mean prediction probability was $P_{K}=0.74$ and this was for RMS power, while for CCSd it was $P_{K}=0.70$. Testing with a multivariate general linear model for differences in prediction probability across the $20 \%, 40 \%$ and $60 \%$ peak gas groups revealed statistically significant differences (Wilks' Lambda: $p=0.003$, F-statistic $=3.628$, partial $\eta^{2}=0.698$ - i.e. effect size). Post-hoc test results for each individual measure found that the CS prediction probability values were statistically significantly different for the $60 \%$ peak gas group as compared to the $20 \%$ and $40 \%$ peak gas groups $(p=0.041)$, and that the RMS power prediction probability values were statistically significantly different for all of the $20 \%, 40 \%$ and $60 \%$ peak gas groups $(p<0.01)$. The other measures did not show statistically significant differences between gas groups. This can be understood given the small number of subjects in each group and the high variability of the prediction probability values across subjects, as is indicated by the standard deviation values in Table 1.

Testing with a repeated measures general linear model revealed statistically significant differences in the prediction probabilities across the different measures for all subjects (the assumption of sphericity was rejected making the use of the Greenhouse-Geisser adjustment necessary: $p=0.03$, F-statistic $(2.191,41.621)=3.69$, partial $\left.\eta^{2}=0.163\right)$. A simple contrast using CCSd as the reference revealed no statistically significant differences between prediction 
probabilities for CCSd and those for CCS, CI, and RMS power, while statistically significant differences were observed between CCSd and CS $(p=0.007)$, pole frequency $(p=0.001)$ and pole damping $\gamma(p=0.001)$. Not enough subjects were available to statistically assess differences in the prediction probabilities for the measures within the different peak gas groups. Measure dependence on concentration for each peak gas group

The dependence of the different measures on gas concentration averaged across individuals is illustrated for each peak gas group in Figure 3. Figures 3(A)-(L) show CS, CCS, CCSd, CI, the frequency of the poles in the $5-15 \mathrm{~Hz}$ band, the damping of the poles in the 5-15 $\mathrm{Hz}$ band, the RMS power of the mean-removed signal, 1-4 Hz ( $\delta$ ) band power, 4-8 $\mathrm{Hz}(\theta)$ band power, 8-15 $\mathrm{Hz}(\alpha)$ band power, $15-30 \mathrm{~Hz}(\beta)$ band power, and 30-110 $\mathrm{Hz}(\gamma$, high $\gamma$ and overlapping broadband EMG) band power, respectively, as a function of gas concentration. Similar to Figure 2, in Figure 3 for the $60 \%$ peak gas group (colored blue) it can be observed that $\mathrm{CS}$ and CCS increase and CCSd and RMS power decrease with increases in $\mathrm{N}_{2} \mathrm{O}$ gas concentration, while CI and pole frequency have only weak correlations with gas level and the pole damping parameter $\gamma$ shows decreases above $40 \%$ gas concentrations although they are not statistically significant based on mixed model testing. For the $20 \%$ (red) and $40 \%$ (green) peak gas group, similar but weaker trends were observed.

Notably as indicated by the stars above the error bars in Figure 3, for the $60 \%$ peak gas group when considering the comparison between the rest and 50-60\% gas concentration bins, mixed model testing for each measure did reveal statistically significant increases in CS (39\%, $p=0.001-$ F-statistic $=3.888$ and $p=0.017$ for test of fixed effects across all gas bins) and $\operatorname{CCS}(42 \%, p=0.002-$ F-statistic $=3.389$ and $p=0.028$ for test of fixed effects across all gas bins) and decreases in CCSd (398\% defined relative to the median resting CCSd value or 34\% defined relative to the median resting CCS value, $p=0.007-$ F-statistic $=2.352$ and $p=0.089$ for test of fixed effects across all gas bins), RMS power $(42 \%, \quad p=0.001-\mathrm{F}-$ 
statistic $=7.664$ and $p=0.001$ for test of fixed effects across all gas bins), $\delta$-band $(1-4 \mathrm{~Hz})$ power $(70 \%, p=0.001-$ F-statistic $=22.2$ and $p=0.001$ for test of fixed effects across all gas bins), $\theta$-band $(4-8 \mathrm{~Hz})$ power $(61 \%, \quad p=0.025-$ F-statistic $=2.38$ and $p=0.097$ for test of fixed effects across all gas bins) and $\alpha$-band $(8-15 \mathrm{~Hz})$ power $(72 \%, \quad p=0.008-$ F-statistic $=2.5$ and $p=0.081$ for test of fixed effects across all gas bins). There was no statistically significant change in the $30-110 \mathrm{~Hz}$ band that includes, $\gamma$, high $\gamma$ and overlapping EMG content. For the $20 \%$ and $40 \%$ peak gas groups and as indicated by the stars above error bars, mixed model testing demonstrated statistically significant differences between rest and gas concentration bins for CCSd, with $p<0.05$ for all comparisons. This is likely due to CCSd being defined as a relative measure normalized by the median resting CCS values which helps to remove the effect of intersubject variability.

Figure 3(N) shows the correlation between gas concentration and aCPT accuracy (i.e. responsiveness) for the group data. Because the 'incorrect' category included missed trials and performance on the aCPT task was high, an incorrect response during the gas recording effectively implies the participant was unresponsive. For high gas levels (above 40\%) it can be seen that a greater proportion of 'incorrect' trials occur implying that high gas concentrations were correlated with significant reductions in responsiveness.

\section{Discussion}

The goal here is not to show that CCSd outperforms measures like CS, CCS or RMS power. Rather the goal here is to show the CCSd gives comparable performance to these measures while at the same time will vary in the same direction during anesthetic-induced reductions in consciousness with either $\mathrm{N}_{2} \mathrm{O}$ or propofol. As discussed below the key findings of this exploratory retrospective study are that CCSd provides a potentially effective measure for tracking changes in sedative $\mathrm{N}_{2} \mathrm{O}$ concentrations using frontal EEG that gives comparable performance to CS, CCS and RMS Power. Moreover, based on previous studies with CS, CCSd 
is expected to also track changes in propofol concentration while also being sensitive to the different potencies of these two anesthetics. This offers the potential to develop a depth of anesthesia monitor that works for different anesthetics with different molecular modes of action.

\section{ARMA measures, $\mathrm{N}_{2} \mathrm{O}$, and Depth of Anesthesia Monitoring}

Determining the sensitivity of the ARMA measures, CS, CCS, CCSd and CI, to different anesthetic agents is important for broadening the scope of anesthetics to which these measures can be applied for depth of anesthesia monitoring. Here it has been shown that high levels of $\mathrm{N}_{2} \mathrm{O}$ (>40\%) that lead to reductions in responsiveness, correspond with small increases in CS and CCS, and a large decrease in CCSd, while a clear conclusion regarding the changes in CI could not be reached. The observed decreases in $\delta, \theta$, and $\alpha$ band power shown in Figure 3 appear to be the underlying EEG frequency changes that drive the changes in CS, CCS and CCSd. CS-based measures are proportional to the magnitude of the mean pole of the ARMA model projected onto the real axis (equations 5-7). Here we compute the mean pole projected onto the real axis over a 2 second window. This measure is one-dimensional and essentially combines the information about the frequency and damping of the dominant oscillations in the EEG over the corresponding 2 second period. Thus the CS-based measures will change with the observed decreases in the $\delta, \theta$, and $\alpha$ band power shown in Figure 3, which correspond to increased damping of these dominant frequencies. From Figure 3 it can also be noted that the lack of statistically significant changes in the $30-110 \mathrm{~Hz}$ band suggests the possibly confounding effects of EMG content have been minimized.

The large standard deviations in the measures for individuals seen in Figure 2, might suggest there are other significant noise effects, however, these fluctuations arise because we are using short EEG analysis windows of 2 seconds in duration to ensure the ARMA models are computed on approximately stationary EEG segments. The more standard approach in depth of anesthesia monitoring is to use larger windows of say 15-30 seconds or compute a moving average of the feature to create a smoother time-varying index of hypnosis [42]. We prefer to 
show the fluctuations seen with the short analysis windows using the error bars and to treat the means for each gas concentration bin as a marker for how a smoothed time-varying index might look when applied in clinical practice.

Here we have focused on the spectral changes linked to CS and CI based features for the typical pre-processing steps for the application of these features as a way of understanding which frequency changes are linked to these features, thus $1-4,4-8,8-15,15-30$ and $30-110 \mathrm{~Hz}$ band powers were calculated on the preprocessed $1-40 \mathrm{~Hz}$ band-pass filtered data before downsampling to $80 \mathrm{~Hz}$ for the CS and CI calculation. (Calculating 30-110 Hz band power on the preprocessed 1-40 $\mathrm{Hz}$ band-pass filtered data still allows for detection of residual EMG and gamma that might be influencing our CS and CI based measures.) Therefore, band power changes should only be considered within this context when comparing them to other studies. Rampil et al. [7] found increases in $40-50 \mathrm{~Hz}$ and $70-110 \mathrm{~Hz}$ band power at $50 \% \mathrm{~N}_{2} \mathrm{O}$ gas concentration as compared to rest, which are outside of the 1-40 Hz band considered here. Yamamura et al. [39] found increases in $30-40 \mathrm{~Hz}$ band power at 30,50 and $70 \% \mathrm{~N}_{2} \mathrm{O}$ gas concentrations and decreases in alpha band power at 50 and $70 \% \mathrm{~N}_{2} \mathrm{O}$ gas concentrations as compared to baseline. There are several differences in the dataset used here and these other studies [7,39], such as gas delivery parameters, electrode positions and referencing schemes. Yamamura et al. [39] indicated that their observed changes in 30-40 $\mathrm{Hz}$ band power were negated by altering the referencing scheme. Here we have used a referencing scheme consistent with the standard application of the CS and CI based measures. Although like Yamamura et al. [39] we see a decrease in alpha band power, we did not see an increase in $30-40 \mathrm{~Hz}$ band power. This suggests our referencing scheme leads to different observed power changes. An alternative possibility is that EMG artefact was present in the Yamamura et al. [39] data in the $30-40 \mathrm{~Hz}$ band, however, this is not likely because Yamamura et al. [39] gave significant consideration to EMG artefact. A true characterisation of the presence of EMG in the EEG during $\mathrm{N}_{2} \mathrm{O}$ inhalation requires further study during neuromuscular blockade [43]. 
Overall these findings are potentially clinically useful as they demonstrate CS, CCS, and CCSd could be used to monitor the level of sedation for adjuvant levels of $\mathrm{N}_{2} \mathrm{O}$ alone prior to general anesthesia, and in conditions where $\mathrm{N}_{2} \mathrm{O}$ is the sole agent. This is significant and important because, as mentioned in the introduction, existing depth of anesthesia monitors do not work reliably with $\mathrm{N}_{2} \mathrm{O}$ [7-13], or other putative NMDA receptor antagonists (in particular ketamine) in general [6], and a new depth of anesthesia monitoring method is needed that can work for all anesthetics, that is independent of its pharmacological mechanism [6].

The increases in CS and CCS of 39\% and 42\%, respectively, and decrease in CCSd of $34 \%$ (defined relative to the absolute value of median resting CCS) for $\mathrm{N}_{2} \mathrm{O}$ at $60 \%$ peak gas levels is consistent with the idea of $\mathrm{N}_{2} \mathrm{O}$ being a weak anesthetic, given that the potent anesthetic propofol previously produced a much larger decrease in CS of $255 \%$ for propofol levels associated with high levels of unresponsiveness (an Observer's Assessment of Alertness/Sedation [44] score of zero) [18]. The magnitude of these changes suggests that CS-derived measures have a graded sensitivity to different levels of unresponsiveness that are induced by anesthetics of differing potency.

The decrease in CCSd of $398 \%$ (defined relative to the absolute value of median resting CCSd) is partly a reflection of the small values for CCSd obtained during rest. This large change arises because the CCSd measure, as defined in Equation 7, involves normalising CCS by the median CCS value during the rest period. Rather than consider percent measure change, the better metric for quantifying how well the measures perform at tracking $\mathrm{N}_{2} \mathrm{O}$ concentration levels is the prediction probability, $P_{K}$. The prediction probability results using the repeated measures general linear model showed that prediction probability for CCSd was statistically significantly different from the prediction probability for CS, with CCSd obtaining a prediction probability of $P_{K}=0.70$ and CS obtaining a lower prediction probability of $P_{K}=0.66$ for the $60 \%$ peak gas group. This suggests CCSd better tracks the level of $\mathrm{N}_{2} \mathrm{O}$ gas concentration than CS and that 
CCSd is more robust to noise. Given that the prediction probability results could not demonstrate that prediction probability for CCSd was statistically significantly different from the prediction probabilities for CCS or RMS power, it is not clear which of these measures is better at tracking $\mathrm{N}_{2} \mathrm{O}$ gas concentration even though CCS and RMS power both had slightly higher prediction probabilities than CCSd for the $60 \%$ peak gas group. Moreover, although RMS power achieved a slightly higher prediction probability than CCSd, it is known that RMS power is effected more by noise, such as electromyographic signals and power changes unrelated to anesthesia, than CSbased measures are [18].

The mean prediction probability value of $P_{K}=0.70$ obtained here for CCSd for the $60 \%$ peak gas group is also consistent with the idea of $\mathrm{N}_{2} \mathrm{O}$ being a weak anesthetic, since more standard depth of anesthesia monitoring measures, such as State Entropy and Response Entropy, have been shown to achieve a higher correlation with anesthetic concentration by demonstrating values of $P_{K}=0.9$ for propofol concentrations spanning the range of participant states from awake to fully anesthetised [42]. It is expected that weaker anesthetics will have less noticeable effects on depth of anesthesia monitoring measures and therefore produce weaker correlations between the measures and the anesthetic concentration. It is very important to note, however, that proper comparison of prediction probabilities for different anesthetics should be performed with the same reference levels (e.g. proportion of minimum alveolar concentration for inhaled anesthetics [45]). Thus, the mean prediction probability value of $P_{K}=0.70$ obtained here for CCSd for the $60 \%$ peak gas group is also consistent with the possibility of CCSd being only a moderate indicator of $\mathrm{N}_{2} \mathrm{O}$ gas concentration, although it performs well compared to the other measures considered here. Future work comparing different anesthetics and EEG measures is needed for clarification.

As mentioned above, in previous work with propofol CS was found to decrease with an increase in anesthetic level [18], while here for $\mathrm{N}_{2} \mathrm{O}$ it was found CS increased. Hence here CCSd 
was devised as a measure that would consistently decrease with increasing levels of propofol and $\mathrm{N}_{2} \mathrm{O}$. Since CCSd is directly related to CS it is expected that decreases in CCSd with increases in propofol concentration will be much larger than the CCSd decreases observed here for $\mathrm{N}_{2} \mathrm{O}$. Future work will be needed to more completely characterize how CS-derived measures and CI vary with different levels of responsiveness induced by different anesthetics.

As is outlined by the pole damping results in Figures 2 and 3 and the Appendix, the changes in CS, CCS, and CCSd for $\mathrm{N}_{2} \mathrm{O}$ can potentially be understood by the increased damping observed for poles in the $5-15 \mathrm{~Hz}$ band. Although the mixed model test for pole damping did not reveal statistically significant differences between rest and the 50-60\% gas concentration bins at the group level, for each subject in the $60 \%$ peak gas group surrogate testing revealed statistically significantly different prediction probabilities for pole damping (mean $P_{K}=0.56$ - indicating a decrease in the pole damping parameter) from the zero-correlation case ( $\left.P_{K}=0.5\right)$. Therefore, the results are inconclusive, however, Figure A1 illustrates that pole damping appeared present for the two subjects who had complete gas recordings in the $60 \%$ peak gas group (Figures A1(B) and (E)). Thus, more data will be needed to make a final conclusion. It is also worth noting that pole damping in the $0-5 \mathrm{~Hz}$ band, not calculated directly here, will also contribute to changes in CS, CCS, and CCSd as can be expected from the observed decreases in $\delta$ and $\theta$ band power.

Similar findings occurred with regards to changes in CI as a function of $\mathrm{N}_{2} \mathrm{O}$ gas concentration. Although the mixed model test for CI did not reveal statistically significant differences between rest and the 50-60\% gas concentration bins at the group level, for three of the four subjects in the $60 \%$ peak gas group surrogate testing revealed statistically significantly different prediction probabilities for CI (mean $P_{K}=0.66$ ) from the zero-correlation case ( $P_{K}=0.5$ ) that also had values greater than 0.5 . This indicates that CI decreased slightly for these three subjects, although for subject 18 the relationship between CI and gas concentration was highly variable (see Figure 2D). On the other hand, for subject 17 in the $60 \%$ peak gas group the 
prediction probability was $P_{K}=0.43$ which corresponds with an increase as is seen in Figure 2D. Therefore, the results for CI are inconclusive. For the combination of the anesthetic propofol and the analgesic remifentanil, CI has been shown to be indicative of the analgesic effects of remifentanil [18]. Determining the utility of CI for tracking $\mathrm{N}_{2} \mathrm{O}$ gas concentration and its analgesic effects will require larger sample sizes and potentially higher gas concentrations, and/or assessment of responsiveness during painful stimuli.

Further consideration of CCSd on larger sample sizes is also likely to be important to verify what has been observed here, primarily for the four subjects in the $60 \%$ peak gas group. For example, in Figure 2 there were some differences in the way CCSd decreased over time for the different subjects. In particular, subject 18 showed a strong drop in CCSd for the $0-10 \% \mathrm{~N}_{2} \mathrm{O}$ gas concentration bin as compared to the higher concentrations indicating that this subject may be more sensitive to the rate of change of delivery of $\mathrm{N}_{2} \mathrm{O}$ than the absolute concentration level. Moreover, across the subjects in Table 1 there is high degree of inter-individual variability in the prediction probability values. Such differences across subjects can only be properly characterised in studies with more subjects. At the same time, however, there are significant challenges in acquiring data at high $\mathrm{N}_{2} \mathrm{O}$ gas concentrations due to risks of nausea, emesis and hypoxia [46]. It is for these reasons that only 4 subjects were studied at the $60 \% \mathrm{~N}_{2} \mathrm{O}$ peak gas concentration level. After the experiences with the first 4 subjects, further study at this level involving delivery of only $\mathrm{N}_{2} \mathrm{O}$ and oxygen was deemed an unethical risk to subjects. Given the difficulties of collecting the data presented here, the data that we do have is of high value. Future studies with modified protocols that include strong antiemetics [47] that reduce nausea and emesis symptoms may be able to yield more data at the $60 \% \mathrm{~N}_{2} \mathrm{O}$ gas concentration level.

Given that the relationship between measures and gas concentration could be nonlinear, for special cases (e.g. CI for the $60 \%$ peak gas group) we applied an exponential transform to a given measure based on a logarithmic fit between the measure and gas concentration. This 
transform was applied to create a linear relationship between the transformed measure and the gas concentration before feeding the transformed measure data into the linear mixed model analysis. However, this did not change the statistical significance results for the mixed model analysis (for brevity results were not included).

Taking into account that CS, CCS and CCSd depend on the mean pole projected onto the real axis, it can be seen that damping causes the mean pole to be closer to the origin and therefore CS, CCS and CCSd change relative to resting state. This change in CS-based measures may be indicative of the weak hypnotic effects of $\mathrm{N}_{2} \mathrm{O}$ [24]. The influence of damping of the dominant oscillations on the CS, CCS and CCSd measures suggests a better characterization of oscillatory damping may be key to understanding ARMA-based CS and CI measure changes for different substances.

Given that $\mathrm{N}_{2} \mathrm{O}$ has both weak anesthetic and strong dissociative effects $[24,48,49]$, it is difficult to disentangle the influence of these effects on the measures and the reductions in responsiveness seen in the subjects for high $\mathrm{N}_{2} \mathrm{O}$ gas levels.

\section{Comparison to other substance combinations}

The combination of $4 \%$ sevoflurane and $66 \% \mathrm{~N}_{2} \mathrm{O}$ has been reported to produce a strong decrease in CI and a slight decrease in CS when compared to $4 \%$ sevoflurane alone [17]. Considering the effects seen here for $\mathrm{N}_{2} \mathrm{O}$ alone, it is therefore apparent that there are significant non-additive effects occurring when sevoflurane is combined with $\mathrm{N}_{2} \mathrm{O}$. Comparing the increase of CS for $\mathrm{N}_{2} \mathrm{O}$ alone (39\%) with the slight decrease of CS (16\%, not statistically significant) for $\mathrm{N}_{2} \mathrm{O}$ in combination with sevoflurane compared to $4 \%$ sevoflurane alone, suggests $\mathrm{N}_{2} \mathrm{O}$ may provide limited additional hypnotic effects when high levels of sevoflurane are already being applied. The lack of observed changes in $\mathrm{CI}$ for $\mathrm{N}_{2} \mathrm{O}$ alone differs from the large (approximately 44\%) decrease in $\mathrm{CI}$ when $66 \% \mathrm{~N}_{2} \mathrm{O}$ is added to $4 \%$ sevoflurane [17]. The mechanisms underlying this difference are not clear. Overall, these points highlight the potential risk that 
for $\mathrm{N}_{2} \mathrm{O}$ in the presence of other anesthetics it will not be possible to detect the same CSbased changes identified here when giving $\mathrm{N}_{2} \mathrm{O}$ alone, and that future work on the study of different anesthetic combinations is required.

A recent study by Pavone et al. [23] looked at the effects on EEG band power observed when high dose $(>60 \%) \mathrm{N}_{2} \mathrm{O}$ gas concentration levels were delivered after sevoflurane-based anesthesia. It was found that strong alpha sub-band $(8-12 \mathrm{~Hz})$ power associated with sevoflurane delivery decreased with replacement with $\mathrm{N}_{2} \mathrm{O}$, and this was also associated with an increase in slow delta band $(0.1-4 \mathrm{~Hz})$ power. The decrease in alpha band power they observed appears consistent with the apparent decrease in $\alpha(8-15 \mathrm{~Hz})$ band power observed here for lower levels of $\mathrm{N}_{2} \mathrm{O}$. It is still somewhat difficult to relate the work of Pavone et al. to the results observed here for $\mathrm{N}_{2} \mathrm{O}$ alone because Pavone et al. applied $\mathrm{N}_{2} \mathrm{O}$ when residual levels of expired sevoflurane were still present and there may be non-additive effects as a result. The strong decrease in alpha band power observed by Pavone et al. could be linked to the strong decrease in CI observed previously for the combination of $4 \%$ sevoflurane and $66 \% \mathrm{~N}_{2} \mathrm{O}$ as compared to sevoflurane alone [17], and the slight decrease in CS for this case could reflect the shift in dominant frequency from alpha band to slow-delta observed by Pavone et al.

\section{Conclusion}

Previous studies with the combinations of propofol and remifentanil [18], and sevoflurane and $\mathrm{N}_{2} \mathrm{O}$ [17], have demonstrated the powerful application of CS and CI to the dissociation of the hypnotic and analgesic effects of these substances. Here it is further demonstrated that CS and CS-derived measures, CCS and CCSd, are sensitive to increases in $\mathrm{N}_{2} \mathrm{O}$ gas concentration and associated induced reductions in responsiveness. This is an important step towards developing depth of anesthesia monitors that are sensitive to putative NMDA receptor antagonist anesthetic-induced reductions in responsiveness and consciousness. It will be 
important to further evaluate CS-based measures and CI using different anesthetics and anesthetic/analgesic combinations in order to more generally evaluate their clinical utility for depth of sedation/anesthesia monitoring. At the same time it will be important to compare the performance of the CS-based and CI measures against other depth of anesthesia monitoring methods, such as the BIS monitor which appears insensitive to increases in $\mathrm{N}_{2} \mathrm{O}$ concentration despite reductions in responsiveness [7,8]. The BIS was not considered here because it is a proprietary method and requires access to a BIS monitor, however, the contributions we present here are useful in the context of the growing body of work on ARMA model-based depth of anesthesia monitoring [16-18,32,50,51]. It will also be important to consider comparisons with methods that take into account the unitary network mechanisms underlying anesthetic-induced loss of consciousness [52] or employ physiologically-motivated neural mass modelling approaches that can infer underlying anesthetic-induced neurophysiological changes [19].

\section{Appendix}

As mentioned in the results, the analysis of frequency and damping of the poles focused on poles with frequencies in the band of 5-15 Hz because Yamamura et al. [39] observed strong effects of $\mathrm{N}_{2} \mathrm{O}$ on damping in this frequency band. Here the effects of $\mathrm{N}_{2} \mathrm{O}$ on the distributions of the poles are demonstrated for individuals for the highest $\mathrm{N}_{2} \mathrm{O}$ gas levels.

Pole histograms of individuals in the $60 \%$ group

For the rest and gas conditions, some of the effects of $\mathrm{N}_{2} \mathrm{O}$ on the EEG can be captured in the pole histograms of the ARMA model which reflect the normalized distributions of the dominant damped oscillations in the EEG. Figure A1(A) partly schematizes the meaning of these pole histograms which are plotted on the complex (number) plane because the poles, $\rho_{k}$, are represented by complex numbers. As described in the methods, the angle of a complex pole with respect to the origin of the complex plane corresponds to the oscillatory frequency of the pole. 
The radial distance of a pole from the origin, reflects the damping of the pole (the closer to the origin the more damped the oscillation). Figures A1(B)-(E) show the pole histograms for the rest (top row) and gas (middle row) data, and the difference between the rest and gas histograms (bottom row) for the four individuals in the $60 \%$ peak gas group. Figures A1(B)-(E) correspond to subjects $17,18,19$ and 20 , respectively. The gas data corresponds to the last five minutes of the equilibrated peak gas period, except for subjects 18 and 19 who, due to nausea and emesis, had their gas recordings shortened to less than 5 and 10 minutes in duration, respectively. For these two subjects the last two minutes of their gas recordings were used. In each subfigure the poles have been histogrammed by binning the complex plane. In the rest and gas data rows, only the portion of the complex plane corresponding to positive frequencies between $0 \mathrm{~Hz}$ (horizontal axis) and $20 \mathrm{~Hz}$ (vertical axis) is shown. In the row highlighting the differences in the rest and gas histograms the region of the complex plane corresponding to the significant alpha subband (8-12 $\mathrm{Hz})$ changes is focused on. The rest data row indicates the presence of a strong alpha $(8-12 \mathrm{~Hz})$ peak during the resting eyes closed condition for all individuals. In the gas data row it can be seen that alpha poles within the same region have become damped. This is also reflected in the row showing the histogram differences where the radially inward blue to red shifts indicate an increase in damping when going from the rest to the gas cases.

\section{Compliance with Ethical Standards}

Conflict of interest: Levin Kuhlmann and David T.J. Liley declare funding support from Swinburne University of Technology intramural funds, ARC Linkage Grant LP120200773, and Cortical Dynamics Ltd, a depth of anesthesia monitoring device company. David T.J. Liley holds an unvalued equity stake in Cortical Dynamics Ltd. 
Ethical approval: All procedures performed in studies involving human participants were in accordance with the ethical standards of the institutional and/or national research committee and with the 1964 Helsinki declaration and its later amendments or comparable ethical standards.

Informed consent: Informed consent was obtained from all individual participants included in the study.

\section{References}

1. Bruhn J, Myles PS, Sneyd R, Struys MM (2006) Depth of anaesthesia monitoring: What's available, what's validated and what's next? Br J Anaesth 97 (1):85-94. doi:ael120 [pii] 10.1093/bja/ael120

2. Myles PS, Leslie K, McNeil J, Forbes A, Chan MT (2004) Bispectral index monitoring to prevent awareness during anaesthesia: The B-Aware randomised controlled trial. Lancet 363 (9423):1757-1763

3. Mashour GA, Shanks AMS, Tremper KK, Kheterpal S, Turner CR, etal. (2012) Prevention of Intraoperative Awareness with Explicit Recall in an Unselected Surgical Population: A Randomized Comparative Effectiveness Trial. Anesthesiology 117 (4):717-725

4. Kettner S (2014) Not too little, not too much: delivering the right amount of anaesthesia during surgery. Cochrane Database of Systematic Reviews 6:10.1002/14651858.ED14000084

5. Punjasawadwong Y, Boonjeungmonkol N, Phongchiewboon A (2014) Bispectral index for improving anaesthetic delivery and postoperative recovery. Cochrane Database of Systematic Reviews 6:CD003843

6. Hirota K (2006) Special cases: Ketamine, nitrous oxide and xenon. Best Practice and Research: Clinical Anaesthesiology 20 (1):69-79

7. Rampil IJ, Kim JS, Lenhardt R, Negishi C, Sessler DI (1998) Bispectral EEG index during nitrous oxide administration. Anesthesiology 89 (3):671-677

8. Barr G, Jakobsson JG, Owall A, Anderson RE (1999) Nitrous oxide does not alter bispectral index: study with nitrous oxide as sole agent and as an adjunct to i.v. anaesthesia. British Journal of Anaesthesia 82 (6):827-830

9. Puri GD (2001) Paradoxical changes in bispectral index during nitrous oxide administration. British Journal of Anaesthesia 86 (1):141-142

10. Hans P, Bonhomme V, Benmansour H, Dewandre PY, Brichant JF, Lamy M (2001) Effect of nitrous oxide on the bispectral index and the $95 \%$ spectral edge frequency of the electroencephalogram during surgery. Anaesthesia 56 (10):999-1002

11. Anderson RE, Barr G, Jakobsson JG (2005) Cerebral state index during anaesthetic induction: a comparative study with propofol or nitrous oxide. Acta Anaesthesiol Scand 49:750-753

12. Anderson RE, Jakobsson JG (2004) Entropy of EEG during anaesthetic induction: a comparative study with propofol or nitrous oxide as sole agent. Br J Anaesth 92 (2):167-170

13. Wong CA, Fragen RJ, Fitzgerald P, McCarthy RJ (2006) A comparison of the SNAP II and BIS XP indices during sevoflurane and nitrous oxide anaesthesia at 1 and $1.5 \mathrm{MAC}$ and at awakening. Br J Anaesth 97 (2):181-186

14. Broersen P (2002) Automatic spectral analysis with time series models. IEEE Trans Instrum Meas 51:211-216 
15. Broersen PMT (2006) Automatic autocorrelation and spectral analysis

16. Liley DT, Cadusch PJ, Gray M, Nathan PJ (2003) Drug-induced modification of the system properties associated with spontaneous human electroencephalographic activity. Phys Rev E Stat Nonlin Soft Matter Phys 68 (5 Pt 1):051906

17. Liley DT, Leslie K, Sinclair NC, Feckie M (2008) Dissociating the effects of nitrous oxide on brain electrical activity using fixed order time series modeling. Comput Biol Med 38 (10):1121-1130. doi:S0010-4825(08)00132-7 [pii] 10.1016/j.compbiomed.2008.08.011

18. Liley DTJ, Sinclair NC, Lipping T, Heyse B, Vereecke HEM, Struys MMRF (2010) Propofol and Remifentanil Differentially Modulate Frontal Electroencephalographic Activity. Anesthesiology 113 (2):292-304

19. Kuhlmann L, Freestone DR, Manton JH, Heyse B, Vereecke HE, Lipping T, Struys MM, Liley DT (2016) Neural mass model-based tracking of anesthetic brain states. NeuroImage 133:438-456

20. Foster B, Liley DTJ (2011) Nitrous oxide paradoxically modulates slow electroencephalogram oscillations: Implications for anesthesia monitoring. Anesth Analg 113:758-765

21. Hopkins PM (2005) Nitrous oxide: a unique drug of continuing importance for anaesthesia. Best Practice \& Research Clinical Anaesthesiology 19 (3):381-389

22. Jevtovic-Todorovi V, Todorovic S, Mennerick S, Powell S, Dikranian K, al. e (1998) Nitrous oxide (laughing gas) is an NMDA antagonist, neuroprotectant and neurotoxin. Nat Med 4:460463

23. Pavone KJ, Akeju O, Sampson A, Ling K, Purdon PL, Brown EN (2016) Nitrous oxideinduced slow and delta oscillations. Clin Neurophysiol 127 (1):556-564

24. Perouansky M, Pearce R, Hemmings Jr H (2010) Inhaled anesthetics: mechanisms of action. In: Miller R (ed) Miller's Anesthesia 7th ed., vol 1. Churchill Livingstone Elsevier, Philadelphia, pp 515-538

25. Alkire M, Hudetz A, Tononi G (2008) Consciousness and anesthesia. Science 322:876-880

26. Foster BL, Liley DT (2012) Effects of nitrous oxide sedation on resting electroencephalogram topography. Clin Neurophysiol 124 (2):417-423

27. Kuhlmann L, Foster BL, Liley DTJ (2013) Modulation of Functional EEG Networks by the $\begin{array}{llllll}\text { NMDA Antagonist Nitrous Oxide. PLoS ONE } 8 & \text { (2):e56434. }\end{array}$ doi:10.1371/journal.pone.0056434

28. Delorme A, Makeig S (2004) EEGLAB: an open source toolbox for analysis of single-trial EEG dynamics including independent component analysis. J Neurosci Methods 134 (1):9-21. doi:10.1016/j.jneumeth.2003.10.009 S0165027003003479 [pii]

29. Bonhomme V, Hans P (2007) Muscle relaxation and depth of anaesthesia: where is the missing link? British journal of anaesthesia 99 (4):456-460

30. Kamata K, Aho A, Hagihira S, Yli-Hankala A, Jäntti V (2011) Frequency band of EMG in anaesthesia monitoring. British journal of anaesthesia 107 (5):822-823

31. Goncharova II, McFarland DJ, Vaughan TM, Wolpaw JR (2003) EMG contamination of EEG: spectral and topographical characteristics. Clinical neurophysiology 114 (9):1580-1593

32. Shoushtarian M, Sahinovic MM, Absalom AR, Kalmar AF, Vereecke HEM, Liley DTJ, Struys MMRF (2016) Comparisons of electroencephalographically derived measures of hypnosis and antinociception in response to standardized stimuli during target-controlled propofolremifentanil anesthesia. Anesth Analg 122 (2):382-392

33. Smith WD, Dutton RC, Smith NT (1996) Measuring the performance of anesthetic depth indicators. Anesthesiology 84 (1):38-51

34. Hogg RV, Ledolter J (1987) Engineering Statistics. MacMillan,

35. Mardia KV, Kent JT, Bibby JM (1979) Multivariate Analysis. Academic Press,

36. Hochberg Y, Tamhane AC (1987) Multiple Comparison Procedures. Wiley, 
37. Kim K, Timm N (2006) Univariate and multivariate general linear models: theory and applications with SAS. CRC Press,

38. Verbeke G, Molenberghs G (2009) Linear mixed models for longitudinal data. Springer Science \& Business Media,

39. Yamamura T, Fukuda M, Takeya H, Goto Y, Furukawa K (1981) Fast oscillatory EEG activity induced by analgesic concentrations of nitrous oxide in man. Anesth Analg 60 (5):283-288

40. Becker DE, Rosenberg M (2008) Nitrous oxide and the inhalation anesthetics. Anesthesia progress 55 (4):124-131

41. Smith WD, Dutton RC, Smith NT (1996) A measure of association for assessing prediction accuracy that is a generalization of non-parametric ROC area. Stat Med 15 (11):1199-1215. doi:10.1002/(SICI)1097-0258(19960615)15:11<1199::AID-SIM218>3.0.CO;2-Y [pii]

10.1002/(SICI)1097-0258(19960615)15:11<1199::AID-SIM218>3.0.CO;2-Y

42. Ferenets R, Vanluchene A, Lipping T, Heyse B, Struys MM (2007) Behavior of entropy/complexity measures of the electroencephalogram during propofol-induced sedation: Dose-dependent effects of remifentanil. Anesthesiology 106 (4):696-706. doi:10.1097/01.anes.0000264790.07231.2d 00000542-200704000-00011 [pii]

43. Schuller P, Newell S, Strickland P, Barry J (2015) Response of bispectral index to neuromuscular block in awake volunteers. British journal of anaesthesia 115 (suppl 1):i95i103

44. Chernik DA, Gillings D, Laine H, Hendler J, Silver JM, Davidson AB, Schwam EM, Siegel JL (1990) Validity and reliability of the Observer's Assessment of Alertness/Sedation Scale: study with intravenous midazolam. J Clin Psychopharmacol 10 (4):244-251

45. Eger EI (2001) Age, minimum alveolar anesthetic concentration, and minimum alveolar anesthetic concentration-awake. Anesthesia \& Analgesia 93 (4):947-953

46. De Vasconcellos K, Sneyd J (2013) Nitrous oxide: are we still in equipoise? A qualitative review of current controversies. British journal of anaesthesia 111 (6):877-885

47. Haft WA, McAffee R (2015) Antiemetics. In: Basic Clinical Anesthesia. Springer, pp 159163

48. Rudolph U, Antkowiak B (2004) Molecular and neuronal substrates for general anaesthetics. Nat Rev Neurosci 5 (9):709-720

49. Brown E, Purdon P, Van Dort C (2011) General anesthesia and altered states of arousal: a systems neuroscience analysis. Annu Rev Neurosci 34:601-628

50. Kuhlmann L, Manton JH, Heyse B, Vereecke HE, Lipping T, Struys MM, Liley DT (2016) Tracking electroencephalographic changes using distributions of linear models: application to propofol-based depth of anesthesia monitoring. IEEE Transactions on Biomedical Engineering:In Press

51. Shoushtarian M, McGlade D, Delacretaz L, Liley D (2015) Evaluation of the Brain Anaesthesia Response Monitor during anaesthesia for cardiac surgery: a double-blind, randomised controlled trial using two doses of fentanyl. Journal of Clinical Monitoring and Computing 30 (6):833-844

52. Lee U, Ku S, Noh G, Baek S, Choi B, Mashour GA (2013) Disruption of frontal-parietal communication by ketamine, propofol, and sevoflurane. Anesthesiology 118 (6):1264-1275 


\section{Table Legends}

Table 1. Prediction probabilities $P_{K}$ for each feature for each individual and the mean in the 20, 40 and $60 \%$ peak gas groups. 


\section{Figure Legends}

Fig. 1 Example frontal (FPz) EEG data from an individual in the $60 \%$ peak gas group (subject 17). EEG data during (A) baseline resting eyes closed and (B) equilibrated peak level of $60 \%$ $\mathrm{N}_{2} \mathrm{O}$ gas concentration. EEG amplitude spectra for (C) baseline and (D) gas.

Fig. 2 Measure dependence on $\mathrm{N}_{2} \mathrm{O}$ gas concentration for individuals in the $60 \%$ peak gas group for (A) CS, (B) CCS, (C) CCSd, (D) CI, (E) the pole frequency $f$ in the $5-15 \mathrm{~Hz}$ band, (F) the pole damping $\gamma$ in the $5-15 \mathrm{~Hz}$ band, and (G) RMS power. In each subfigure the mean (solid dots) and standard deviation (error bars) of a given measure for each rest and $\mathrm{N}_{2} \mathrm{O}$ gas concentration bin are shown for each individual in the $60 \%$ peak gas group. $(\mathrm{H})$ Legend coding each individual in the $60 \%$ peak gas group - subjects 17 (red), 18 (green), 19 (blue) and 20 (purple) - and the number of epochs, $\mathrm{n}$, contributing to each mean and error bar for each individual. The * occurring above the error bars for the rest bin for each individual indicate statistical significance of the surrogate-based Mann-Whitney $U$ test for the comparison of prediction probabilities for a given measure with the prediction probabilities for its associated zero-correlation (i.e. $P_{K}=0.5$ ) surrogates for $p<0.01$.

Fig. 3 Measure dependence on $\mathrm{N}_{2} \mathrm{O}$ gas concentration and aCPT accuracy for the 20, 40 and $60 \%$ peak gas groups. (A) CS, (B) CCS, (C) CCSd, (D) CI, (E) the pole frequency $f$ in the $5-15 \mathrm{~Hz}$ band, (F) the pole damping $\gamma$ in the 5-15 Hz band, $(\mathrm{G})$ RMS power, $(\mathrm{H}) 1-4 \mathrm{~Hz}(\delta)$ band power, (I) 4-8 Hz ( $\theta$ ) band power, (J) 8-15 Hz ( $\alpha$ ) band power, $(\mathrm{K})$ 15-30 Hz ( $\beta$ ) band power, and (L) 30$110 \mathrm{~Hz}(\gamma$, high $\gamma$ and overlapping broadband $\mathrm{EMG})$ band power as a function of $\mathrm{N}_{2} \mathrm{O}$ gas concentration for each gas group. In (A)-(L) the group mean (solid dots) and standard deviation (error bars) of a given measure for each rest and $\mathrm{N}_{2} \mathrm{O}$ gas concentration bin are shown for each peak gas group. (M) Legend coding each $\mathrm{N}_{2} \mathrm{O}$ peak gas group $-20 \%$ (red), $40 \%$ (green), and $60 \%$ (blue) - and the number of subjects, $n$, contributing to each mean and error bar for each group. The $*$ and $* *$ occurring above the error bars for the different gas concentration bins for 
each group indicate statistical significance of the mixed model testing for the comparison of the measure values at rest and in the corresponding gas concentration bin for $p<0.05$ and $p<0.01$, respectively. (N) The relationship between gas concentration and aCPT accuracy (responsiveness). In $(\mathrm{N})$ the legend indicates the peak gas group: $20 \%$ (red), $40 \%$ (green), and $60 \%$ (blue). Each bar represents the probability that a given gas concentration range was correlated with either an 'incorrect' (top row) or 'correct' (bottom row) response.

Fig. A1 Normalised pole histograms for individuals in the $60 \%$ peak gas group. (A) Schematic illustrating the relationship between pole frequency and damping in the complex plane. An increase in pole angle coincides with an increase in pole frequency and thus the frequency of the dominant oscillations in the EEG. A decrease in pole radius, $\boldsymbol{\gamma}$, means an increase in pole damping and thus a decrease in amplitude of the dominant oscillations of the EEG. (B)-(E) The pole histograms for the rest (top row) and gas (middle row) data, and the subtraction of the gas histogram from the rest histogram zoomed in at the alpha band region (bottom row). (B)-(E) correspond to the same participants in Figure 2(A)-(D), respectively. The color bars on the right of the gas histogram code the probability of a pole occurring at a particular point in the complex plane and apply to both the rest and gas histograms of the corresponding individual. The color bars to the right of the difference between the histograms codes the probability difference between the two normalized histograms. Blue, white and red indicate where the rest histogram is greater than, equal to, and less than the gas histogram, respectively. 
Tables

Table 1. Prediction probabilities $P_{K}$ for each feature for each individual and the mean in the 20, 40 and $60 \%$ peak gas groups.

\begin{tabular}{|c|c|c|c|c|c|c|c|}
\hline Feature & $\mathrm{CS}$ & CCS & CCSd & CI & Freq. & $\gamma$ & Power \\
\hline \multicolumn{8}{|c|}{$20 \%$ peak gas group } \\
\hline Subject 1 & 0.56 & 0.65 & 0.62 & 0.44 & 0.50 & 0.48 & 0.48 \\
\hline Subject 2 & 0.64 & 0.81 & 0.81 & 0.22 & 0.50 & 0.47 & 0.44 \\
\hline Subject 3 & 0.54 & 0.55 & 0.55 & 0.38 & 0.60 & 0.47 & 0.55 \\
\hline Subject 4 & 0.51 & 0.53 & 0.51 & 0.62 & 0.48 & 0.53 & 0.57 \\
\hline Subject 5 & 0.45 & 0.48 & 0.53 & 0.50 & 0.55 & 0.46 & 0.47 \\
\hline Subject 6 & 0.48 & 0.42 & 0.58 & 0.52 & 0.51 & 0.51 & 0.48 \\
\hline Subject 7 & 0.53 & 0.54 & 0.53 & 0.47 & 0.51 & 0.53 & 0.51 \\
\hline Subject 8 & 0.42 & 0.39 & 0.59 & 0.60 & 0.48 & 0.46 & 0.47 \\
\hline Mean & 0.52 & 0.55 & 0.59 & 0.47 & 0.52 & 0.49 & 0.50 \\
\hline Std & 0.06 & 0.13 & 0.09 & 0.12 & 0.04 & 0.03 & 0.04 \\
\hline \multicolumn{8}{|c|}{ 40\% peak gas group } \\
\hline Subject 9 & 0.69 & 0.75 & 0.76 & 0.35 & 0.43 & 0.65 & 0.54 \\
\hline Subject 10 & 0.50 & 0.54 & 0.51 & 0.60 & 0.56 & 0.51 & 0.55 \\
\hline Subject 11 & 0.54 & 0.56 & 0.54 & 0.71 & 0.57 & 0.47 & 0.67 \\
\hline Subject 12 & 0.45 & 0.44 & 0.49 & 0.63 & 0.54 & 0.45 & 0.59 \\
\hline Subject 13 & 0.49 & 0.53 & 0.52 & 0.69 & 0.47 & 0.47 & 0.66 \\
\hline Subject 14 & 0.30 & 0.19 & 0.79 & 0.82 & 0.51 & 0.36 & 0.66 \\
\hline Subject 15 & 0.62 & 0.74 & 0.69 & 0.51 & 0.35 & 0.48 & 0.65 \\
\hline Subject 16 & 0.56 & 0.63 & 0.61 & 0.66 & 0.42 & 0.46 & 0.63 \\
\hline Mean & 0.52 & 0.55 & 0.61 & 0.62 & 0.48 & 0.48 & 0.62 \\
\hline Std & 0.11 & 0.17 & 0.11 & 0.13 & 0.07 & 0.08 & 0.05 \\
\hline \multicolumn{8}{|c|}{$60 \%$ peak gas group } \\
\hline Subject 17 & 0.79 & 0.78 & 0.77 & 0.43 & 0.47 & 0.54 & 0.77 \\
\hline Subject 18 & 0.54 & 0.59 & 0.58 & 0.64 & 0.55 & 0.52 & 0.70 \\
\hline Subject 19 & 0.68 & 0.73 & 0.71 & 0.57 & 0.60 & 0.55 & 0.68 \\
\hline Subject 20 & 0.66 & 0.78 & 0.74 & 0.76 & 0.42 & 0.62 & 0.82 \\
\hline Mean & 0.66 & 0.72 & 0.70 & 0.60 & 0.51 & 0.56 & 0.74 \\
\hline Std & 0.09 & 0.08 & 0.07 & 0.12 & 0.07 & 0.04 & 0.06 \\
\hline
\end{tabular}

Std: Standard deviation. Bold Italic $P_{K}$ values indicate statistically significant different from zero correlation based on surrogate testing $(\mathrm{p}<0.01)$ and values above 0.5 . Bold $P_{K}$ values indicate the mean value within each group. CS: cortical state, CCS: composite cortical state, CCSd: composite cortical state distance, CI: cortical input, Freq.: Pole frequency, $\boldsymbol{\gamma}$ : pole damping, Power: RMS power. 
Figures
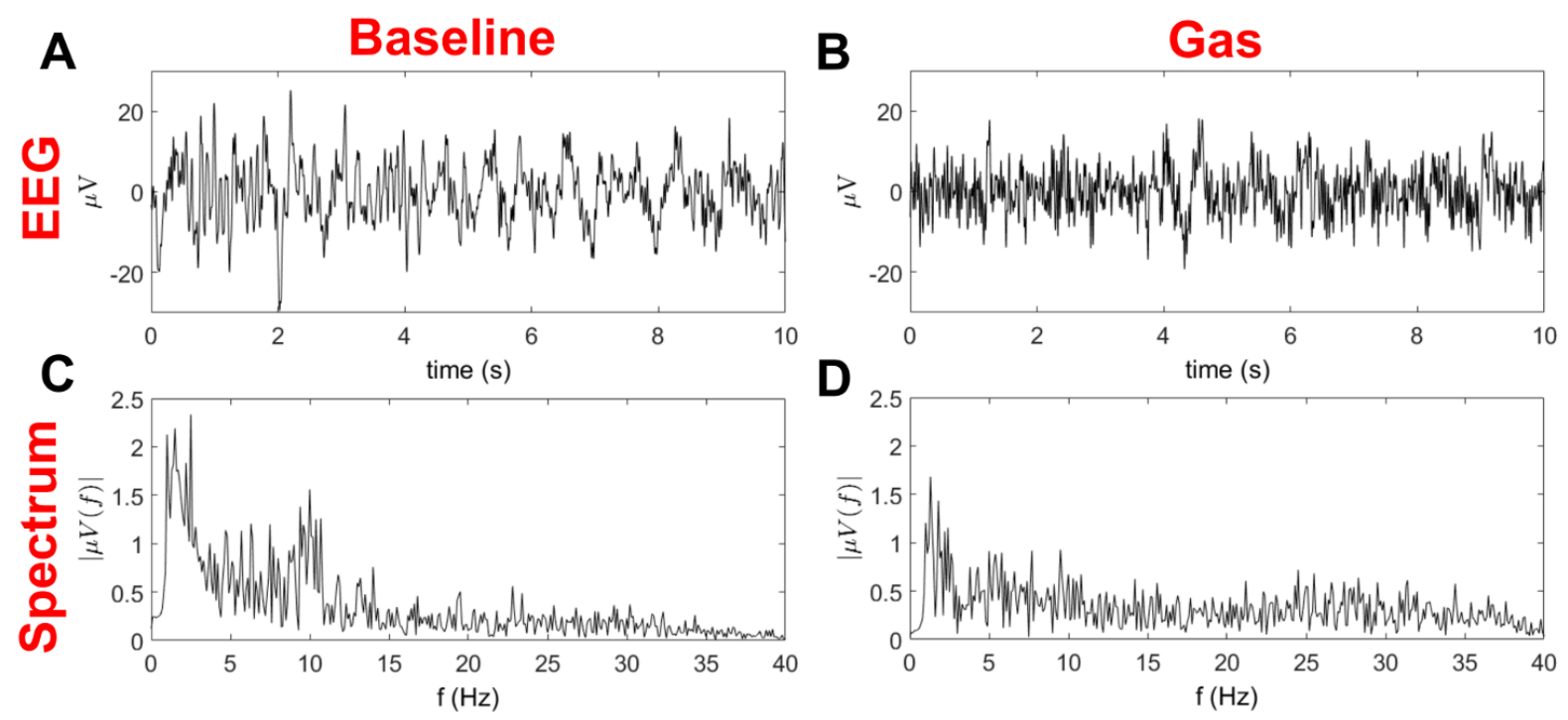

Fig. 1 


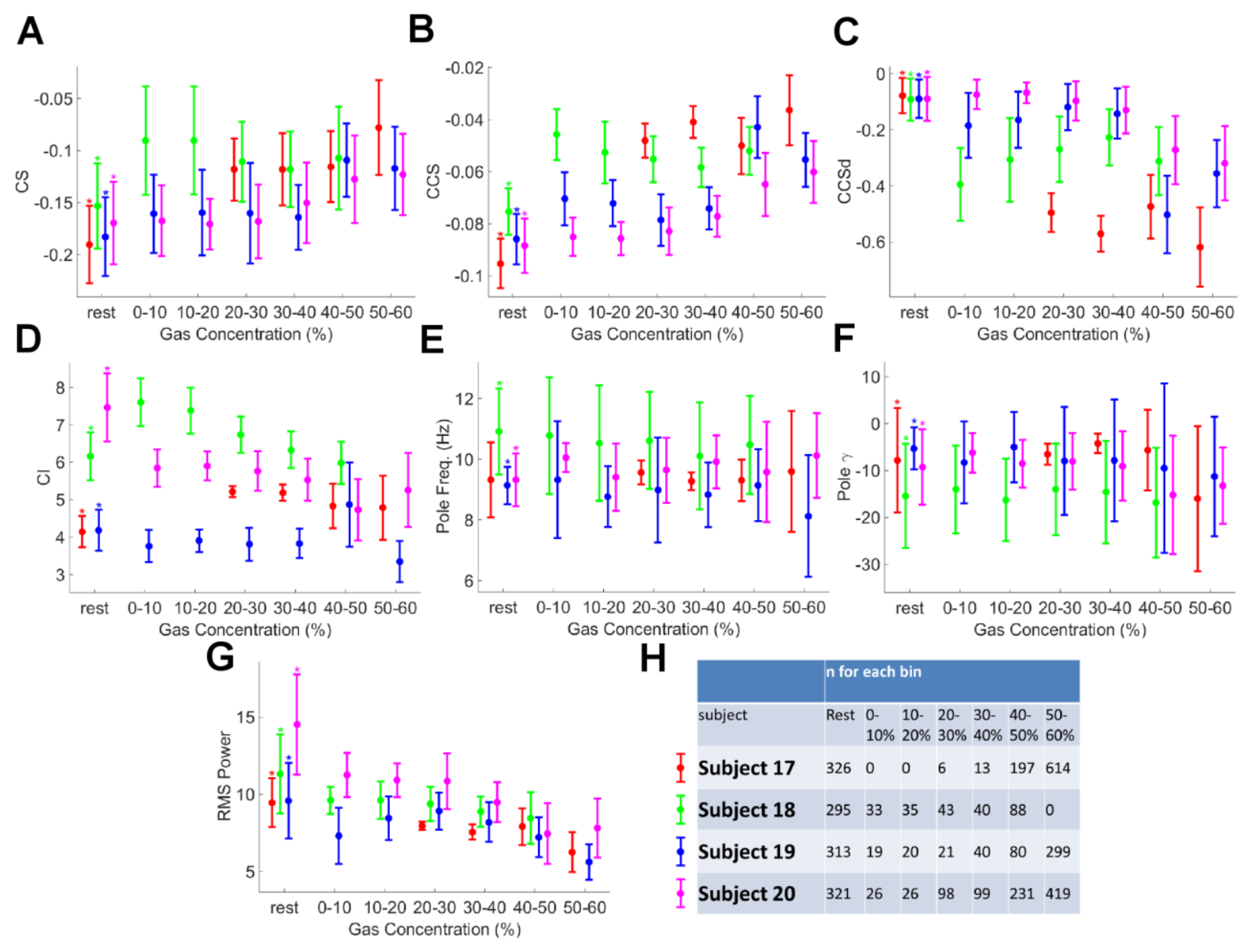

Fig. 2 


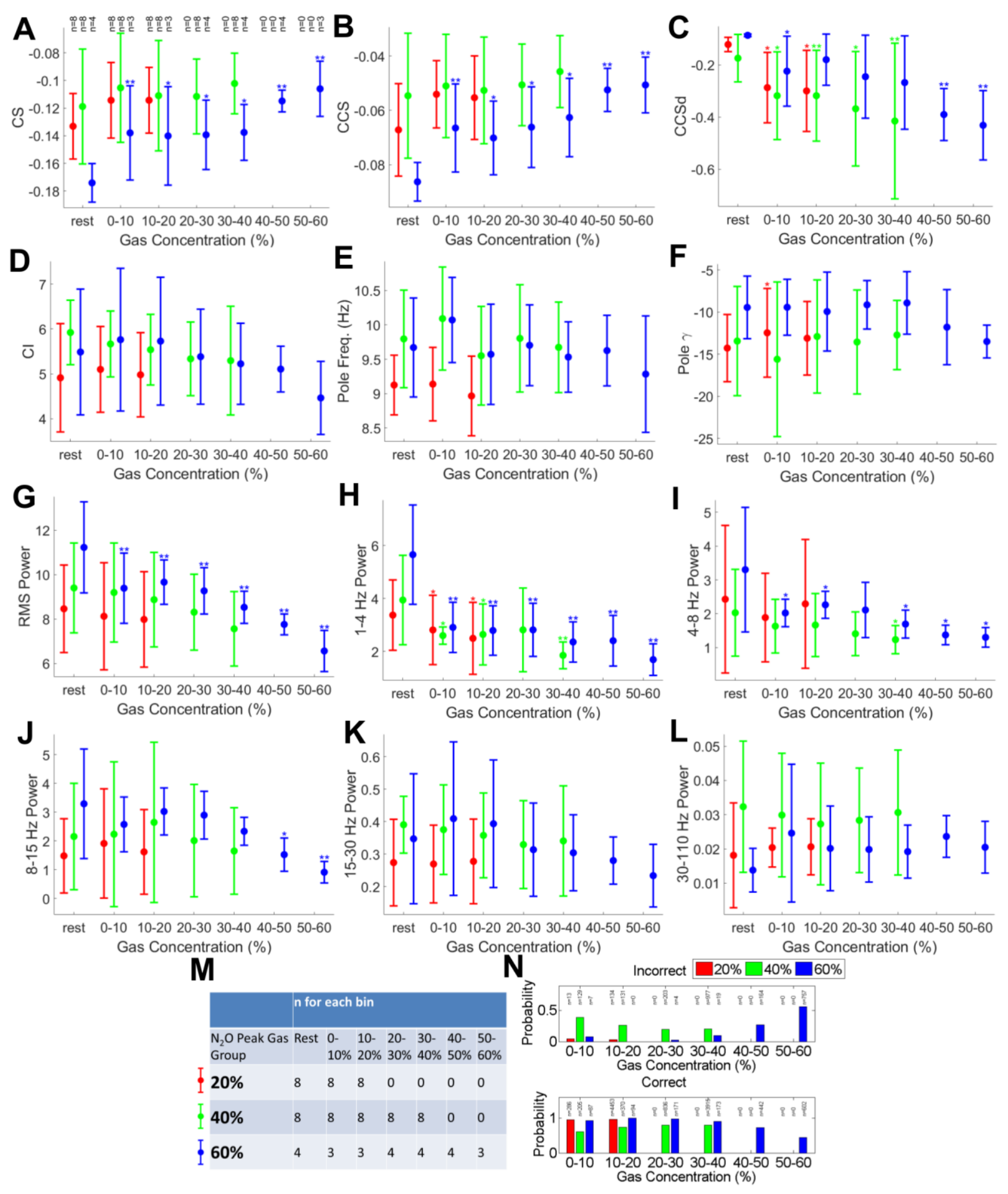

Fig. 3 

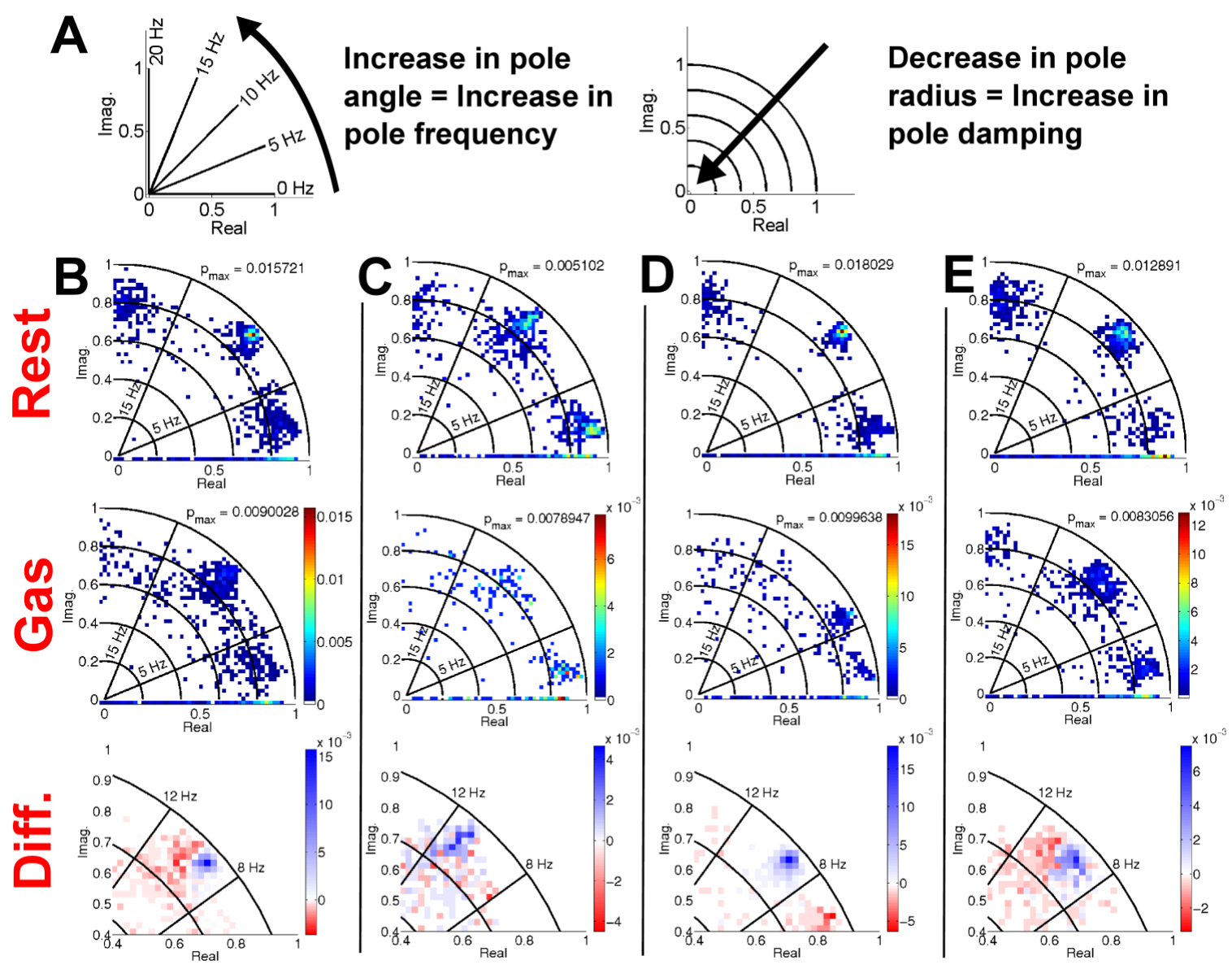

Fig. A1 